\title{
Modeling the Structure of the Windy Torus in Quasars
}

\author{
Sarah C. Gallagher, ${ }^{1}$ Mathew M. Abado ${ }^{1}$ and John E. Everett ${ }^{2}$ \\ ${ }^{1}$ University of Western Ontario, London, ON Canada \\ email: sgalla4@uwo.ca \\ ${ }^{2}$ Northwestern University, Evanston, IL, USA
}

\begin{abstract}
Mass ejection in the form of winds or jets appears to be as fundamental to quasar activity as accretion. A convincing argument for radiation pressure driving this ionized outflow can be made within the dust sublimation radius. Beyond, radiation pressure is even more ubiquitous, as high energy photons from the central engine can now push on dust grains. This physics underlies the dusty-wind model for the putative obscuring torus. Specifically, the dusty wind in our model is first launched from the outer accretion disk as a magneto-centrifugal wind and then accelerated and shaped by radiation pressure from the central continuum. Such a wind can plausibly account for both the necessary obscuring medium to explain the observed ratio of broad-to-narrow-line quasars and the mid-infrared emission commonly seen in quasar spectral energy distributions.
\end{abstract}

Keywords. galaxies: active, quasars: general, infrared: galaxies

\section{Introduction}

Researchers currently studying active galactic nuclei agree on a few key premises. The central engine is powered by a supermassive black hole, and the optical through ultraviolet continuum emission is generated by optically thick material in an accretion disk within the central parsec down to a few gravitational radii. The broad-line region that gives rise to the strong emission features evident in UV and optical quasar spectra is gas illuminated by the accretion disk continuum that is moving within the gravitational sphere of influence of the supermassive black hole. In many luminous quasars, the broad UV emission lines show signatures of winds. The most obvious are the P Cygni profiles seen in the high ionization resonance lines of Broad Absorption Line (BAL) quasars (e.g., Weymann et al. 1991). More subtle signatures are the blue-shifts and asymmetries seen in the same lines in other luminous quasars (e.g., Richards et al. 2011). The wind manifest in these lines is understood to be driven by radiation pressure on ions at UV resonance transitions (e.g., Murray et al. 1995).

One structure that is more uncertain is the so-called torus. The inner radius of the torus is set by the dust sublimation radius, beyond which some fraction of the accretion disk continuum is absorbed by grains and reradiated. Operationally, the torus must account for the $\sim 30 \%$ of the integrated radiant quasar power that comes out in the near-to-midinfrared. Furthermore, it must obscure the central continuum and broad-line region in a significant fraction of Type 2 (narrow-line) objects. While a static toroidal structure (whether smooth or clumpy) beyond the dust sublimation radius will serve these purposes (e.g., Nenkova et al. 2008), such a cold (100-1500 K) structure with the dust mass implied by the mid-infrared luminosity would quickly become gravitationally unstable and collapse. This is an old problem that was addressed by Königl \& Kartje (1994) with a magneto-hydrodynamically launched wind shaped also by radiation pressure on dust. 
This picture was expanded by Keating et al. (2012; hereafter K12) by incorporating dust-radiation driving into the magneto-hydrodynamic (MHD) wind models of Everett (2005), and then illuminating the wind with the central continuum to generate a model spectral energy distribution (SED). While the details of the shape of the output continuum still require some refining to match those observed, the overall power and general shape generated by the K12 'fiducial' model is promising. This model uses as inputs the empirical SDSS composite quasar continuum (Richards et al. 2006), a black hole mass of $M_{\mathrm{BH}}=10^{8} \mathrm{M}_{\odot}$, an Eddington ratio of $L / L_{\mathrm{Edd}}=0.1$, and a column density at the base of the wind of $N_{\mathrm{H}, 0}=10^{25} \mathrm{~cm}^{-2}$. We further assume a Milky Way interstellar-medium dust distribution (Draine \& Li 2007). In this article, we expand on the results presented in K12 by comparing the predictions of the wind model against observations of obscured and unobscured quasar populations.

\section{The Dusty Wind Model}

Following Blandford \& Payne (1982) and Königl \& Kartje (1994), we model the torus as a self-similar dusty wind driven by MHD forces and radiation pressure. The MHD radiative wind code has been described in more detail previously (Everett 2005; K12), and we give only a brief outline here. First, a purely MHD-driven wind solution is calculated given a set of initial conditions input by the user. Next, CLOUDY (version 06.02.09b, as described by Ferland et al. 1998) is called to calculate the radiation field and dust opacity in the wind, allowing for the model to determine the radiative acceleration of the wind due to the central accretion disk continuum. A new MHD solution is calculated for the wind, now taking into account the radiative acceleration, and the process is iterated until the wind converges to a stable profile. Once the wind profile is set, we can investigate the properties of the wind from the data generated by the model, including the density distribution, line-of-sight column densities for important ions, and the covering fraction of the wind.

\section{Broad vs. Narrow Line AGN}

The column densities of 30 elements at each ionization state as well as those of several important molecules are calculated by CLOUDY in the course of the wind simulation. Because they are part of a continuous wind, the total column densities are smooth functions of inclination angle, monotonically increasing toward the plane of the disk (where the polar angle $\theta=90^{\circ}$ ). Therefore, they offer no obvious inclination angle to choose as the boundary for the wind, and a plot of column density versus height does not show any obvious features that define a 'height' for the wind. Also available from CLOUDY is the cross section per proton for the dust in the wind as a function of photon energy. When multiplied by the total column density of hydrogen (i.e., both neutral and ionized), this serves as a measure of the optical depth of the wind. Figure 1 shows the optical depth as a function of polar angle $\theta$ for a few selected wavelengths. This optical depth is also a smooth function of $\theta$ for a wide range of wavelengths, and similarly provides no obvious angle to mark the boundary of the wind. We have therefore chosen the angle at which the dust reaches an optical depth of 5 for light at a wavelength of $6563 \AA$ (the wavelength of $\mathrm{H} \alpha$ emission) to be a reasonable definition of the opening angle of the dusty wind. At this value of $\tau$, only $0.7 \%$ of the broad $\mathrm{H} \alpha$ emission is transmitted through the wind, and so a quasar viewed from this inclination angle (or closer to the disk) would be classified as an optical narrow-line object. 


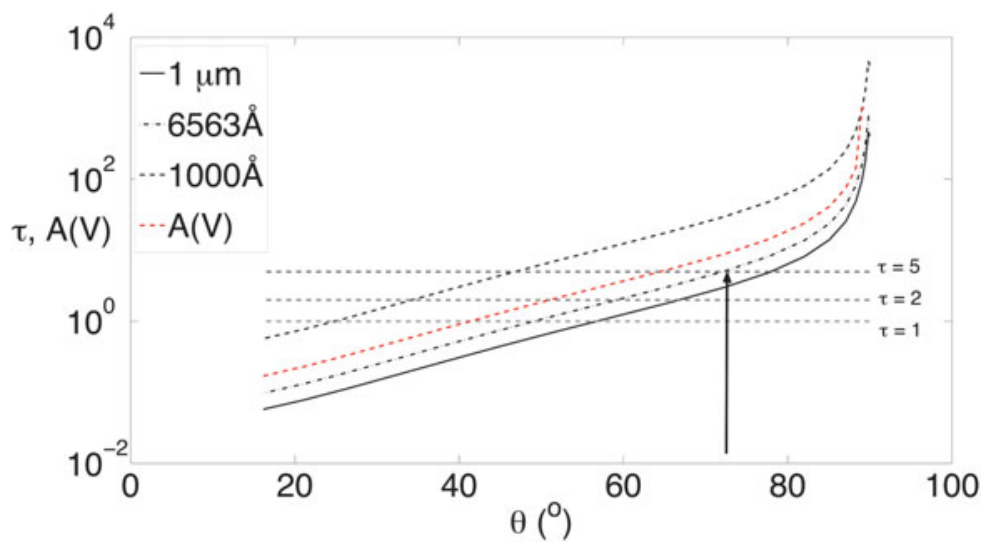

Figure 1. The optical depth at three characteristic wavelengths $(1 \mu \mathrm{m}, 6563 \AA$, and $1000 \AA)$ through the dusty wind plotted vs. inclination angle, $\theta$, where $\theta=90^{\circ}$ is in the plane of the disk. Shown here is the fiducial model, with $N_{\mathrm{H}, 0}=10^{25} \mathrm{~cm}^{-2}, L / L_{\mathrm{Edd}}=0.1$, and $M_{\mathrm{BH}}=10^{8} \mathrm{M}_{\odot}$. The grey dashed lines indicate $\tau$ values of 1,2 , and 5 as labeled. The angle at which $\tau_{6563 \AA}=5$ (marked with a vertical black line) defines the broad-line/narrow-line boundary at $\theta=73^{\circ}$. The red dashed curve just above the optical depth curve at $6563 \AA$ is the $V$-band extinction; $A_{V}$ has a value of 7.7 for $\tau_{\mathrm{H} \alpha}=5$.

For the suite of models run by K12, typical opening angles using this criterion are $\sim 70^{\circ}$, which lead to Type 2 fractions of $\sim 30 \%$ (or Type 2/Type $1 \sim 43 \%$ ). In their survey of low-redshift, emission-line galaxies from the SDSS, Hao et al. (2005) found that Type 1 objects outnumber Type 2 objects by a factor of $2-4$ at high luminosities. A similar study by Simpson et al. (2005) found Type 1 fractions of $\sim 60 \%$ at the luminous end (in terms of [O III] luminosity). Both are generally consistent with our results. Note however, that a Type 2 fraction that decreases with increasing luminosity does not follow naturally from our model, as the wind covering fraction is not sensitive to luminosity directly (for the parameters we have tested). It should be kept in mind, however, that the host galaxy can also serve as a significant source of obscuration of the broad-line region. If accretion disks are randomly oriented with respect to their host galaxies as expected, the observed Type 2 fractions are inflated with respect to the torus-covering fraction because of lineof-sight obscuration occurring from optically thick dust within the host galaxy. Since the size of the broad-line region scales with luminosity $\left(\propto L^{0.5}\right.$; Kaspi et al. 2000; Bentz et al. 2009), more luminous broad line regions are larger and therefore harder to hide. Furthermore, weak broad lines from low-luminosity objects can be "hidden" by a strong stellar continuum (Moran et al. 2002; Martini et al. 2006); detecting these requires high $\mathrm{S} / \mathrm{N}$ spectra and careful modeling of the stellar galaxy emission (e.g., Ho et al. 1997; Hao et al. 2005).

The modeled range of opening angles is tight, and shows little dependence on model input parameters with the exception of the column density at the base of the wind, and the inner wind launch radius. This can be seen in Figure 2, where there is a clear trend relating the opening angle and the base column density with relatively little spread among the suite of models from K12.

Here we are defining Type 1/Type 2 as broad-line/narrow-line with respect to $\mathrm{H} \alpha$, however, the X-ray community often uses the detection of intrinsic X-ray absorption with $N_{\mathrm{H}} \geqslant 10^{22} \mathrm{~cm}^{-2}$ to distinguish obscured from unobscured quasars. The two criteria overlap, but do not define identical populations, as dust extinction and $\mathrm{H}$ I gas absorption do not always go hand-in-hand. The $N_{\mathrm{H}}$ value at what we have defined as our Type $1 / 2$ 


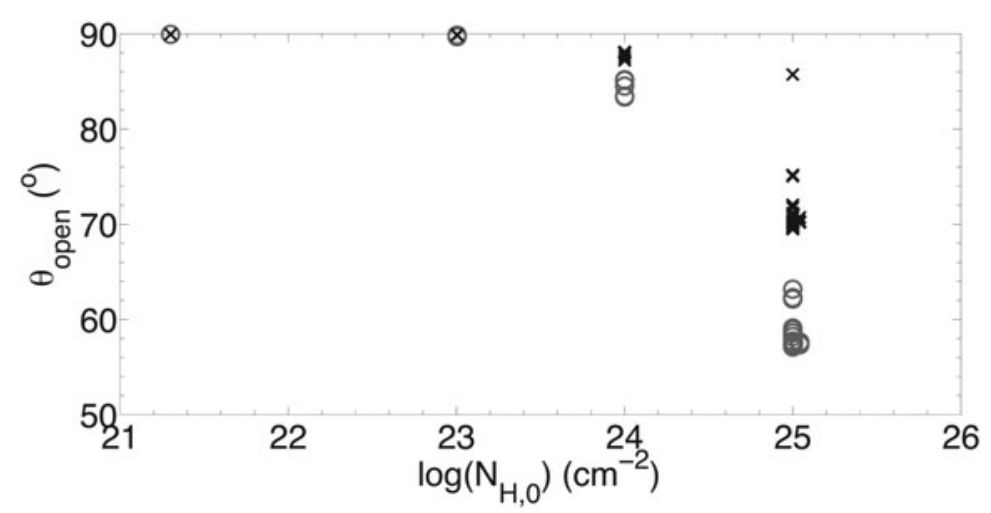

Figure 2. The opening angle, $\theta_{\mathrm{open}}$, as a function of column density, $N_{\mathrm{H}, 0}$ at the base of the wind. At small base column densities, the opening angle is implausibly large. For $N_{\mathrm{H}, 0}=10^{25} \mathrm{~cm}^{-2}$, the values drop to $\sim 70^{\circ}$. This is also the value of $N_{\mathrm{H}, 0}$ that generates appropriate nearto-mid-infrared power in the SED (K12). The black crosses denote the angle where the wind reaches an optical depth of 5 to the broad $\mathrm{H} \alpha$ emission line. The grey circles show the angles at which the same models reach $\tau_{6563 \AA} \stackrel{\circ}{=}$. The models are those presented in K12.

boundary is $\sim 10^{22} \mathrm{~cm}^{-2}$, and therefore consistent with both the X-ray and optical definitions. In the wind model, this also sets a floor for the amount of intrinsic absorption that would be found in optical Type 2 objects, consistent with the observations of X-ray occultation events by Markowitz et al. (2013).

\section{Conclusions}

In a typical cartoon of the structure of an AGN, the so-called torus serves the function of generating the near-to-mid infrared continuum emission in the SED and blocking the direct view of the accretion disk and broad-line region in some fraction of AGN. Both of these roles can be performed by an MHD wind in which radiation driving on dust is crucial. Further comparisons with observations are needed to see if this picture can overturn the torus paradigm.

\section{References}

Bentz M. C., Peterson B. M., Netzer H., Pogge R. W., \& Vestergaard M., 2009, ApJ, 697, 160 Blandford R. D. \& Payne D. G., 1982, MNRAS, 199, 883

Draine B. T. \& Li A., 2007, ApJ, 657, 810

Everett J. E. 2005, ApJ, 631, 689

Ferland G. J., et al. 1998, PASP, 110, 761

Hao L., et al. 2005, AJ, 129, 1795

Ho L. C., Filippenko A. V., Sargent W. L. W., \& Peng C. Y., 1997, ApJS, 112, 391

Kaspi S., Smith P. S., Netzer H., Maoz D., Jannuzi B. T., \& Giveon U., 2000, ApJ, 533, 631

Keating S. K., Everett J. E., Gallagher S. C., \& Deo R. P., 2012, ApJ, 749, 32

Königl A. \& Kartje J. F., 1994, ApJ, 434, 446

Markowitz, A., Krumpe, M., \& Nikutta, R. 2013, AAS/HEAD, 13, \#108.10

Martini P., Kelson D. D., Kim E., Mulchaey J. S., \& Athey A. A., 2006, ApJ, 644, 116

Murray N., Chiang J., Grossman S. A., \& Voit G. M., 1995, ApJ, 451, 498

Nenkova, M., Sirocky, M. M., Nikutta, R., Ivezić, Ž., \& Elitzur, M. 2008, ApJ, 685, 160

Richards G. T., et al. 2011, AJ, 141, 167

Richards G. T., et al. 2006, ApJS, 166, 470

Simpson C. 2005, MNRAS, 360, 565

Weymann R. J., Morris S. L., Foltz C. B., \& Hewett P. C., 1991, ApJ, 373, 23 